\title{
Improvement in Radicular Symptoms but Continued Facet Arthropathy and Axial Back Pain Following Rupture of a Facet Joint Synovial Cyst
}

\author{
Bryan J. Kratz, Troy Buck, Daniel Cramer \\ Department of Pain Management, Loyola University Medical Center, Maywood, IL, USA \\ Email: Bryan.Kratz@lumc.edu
}

How to cite this paper: Kratz, B.J., Buck, T. and Cramer, D. (2018) Improvement in Radicular Symptoms but Continued Facet Arthropathy and Axial Back Pain Following Rupture of a Facet Joint Synovial Cyst. Neuroscience \& Medicine, 9, 46-52. https://doi.org/10.4236/nm.2018.91006

Received: December 13, 2017

Accepted: March 20, 2018

Published: March 23, 2018

Copyright $\odot 2018$ by authors and Scientific Research Publishing Inc. This work is licensed under the Creative Commons Attribution International License (CC BY 4.0).

http://creativecommons.org/licenses/by/4.0/

\begin{abstract}
Lumbar synovial cysts are benign fluid collections thought to form in a background of facet joint degeneration, allowing for fluid to leak from the joint capsule and form cysts in the synovium. Although often asymptomatic, patients with symptomatic synovial cysts will present with low back pain and possibly an associated radiculopathy. Clinicians can consider conservative management, epidural steroid injection, surgical intervention, or facet joint block with aspiration and rupture. This case describes a 59-year-old male facilities manager with intermittent low back pain for one year with worsening right-sided radicular symptoms secondary to a lumbar facet joint synovial cyst in the context of severe facet arthropathy and microinstability. The patient's low back pain and radicular symptoms were refractory to conservative treatment. Imaging demonstrated a lumbar synovial cyst and subsequent management included transforaminal epidural steroid injection and facet joint block with cyst aspiration and rupture. The patient's radicular pain resolved but axial lumbar pain returned after 3 weeks of relief. Follow-up imaging demonstrated decreased cyst size with fluid accumulation and joint space widening. Although the cyst was successfully decompressed with resolution of radicular pain, the underlying facet arthropathy remains contributing to persistent axial low back pain and potential for continued degenerative changes including cyst recurrence.
\end{abstract}

\section{Keywords}

Facet Joint Synovial Cyst, Lumbar Synovial Cyst, Radicular Pain, Cyst Rupture, Low Back Pain, Non-Surgical Management 


\section{Introduction}

Lumbar synovial cysts (LSC) are benign fluid collections arising from the zygapophyseal joint capsule of the lumbar spine. There is a deficiency in the literature on their etiology, prevalence, evaluation, and management [1].

LSC are thought to form when facet joint (FJ) degeneration allows for fluid build-up in the joint, which escapes the joint capsule and forms cysts in the synovium [2] [3] [4] [5] [6]. Symptomatic LSC are most often found posterior and lateral to the dural sac, anterior to the FJ [6]. Obstruction of the spinal canal or neuraxial foramen can lead to symptoms.

Symptomatic LSC are generally 3 - $12 \mathrm{~mm}$ in diameter [6]. Prevalence is unclear, but they are more common in females, typically in the 6th decade of life [1]. The diagnosis of LSC has become more common with the rise in availability and use of high resolution MRI. Patients generally present with symptoms of low back pain (LBP), disc herniation, and radicular pain. Symptomatic LSC are most common at L4-L5 (68\%) followed by L5-S1 (28\%) [1].

At each vertebral level, two FJ and one intervertebral disc articulate together to form a "motion segment" of the spine [7]. The FJ provides a posterior load bearing support, as well as stabilization of the motion segment in flexion, extension, and axial rotation [8]. Spinal muscles supply proprioceptive feedback and stabilize the spinal column to minimize load transmission [7]. The FJ orientation varies by level of the spine and load bearing is largely dependent on spinal posture, among other factors [7]. Facet arthropathy can occur due to poor load distribution, joint misalignment, and failure of the motion segment, including degenerative scoliosis and spondylolithesis [7] [8]. Pain is caused by damage to the heavily innervated joint capsule, synovium, and subchrondral bone. Degenerative changes lead to cartilage degradation, joint space narrowing, and subchrondral bone sclerosis [8]. Risk factors for FJ arthropathy include age, obesity, physical trauma, occupational factors, sex, and smoking [8].

Potential management options include conservative treatment, steroid injection, and cyst aspiration or rupture, and surgical treatment. FJ steroid injection with cyst rupture is a less invasive option that will provide analgesia with the effect of the steroid and decrease pressure on surrounding anatomy with cyst rupture [9]. Studies have shown that $46 \%$ of patients treated with steroid injection and attempted cyst rupture will avoid subsequent back surgery [1].

\section{Case Report}

This case report describes a patient having severe facet arthropathy with associated radicular pain attributed to a facet joint synovial cyst. Our patient is a 59 year old male facilities manager with intermittent LBP for one year. On initial consultation he presented with progressive lower lumbar pain worse with standing up that now had started to radiate down his right buttock with occasional tingling in his foot. Physical exam elicited pain with flexion, extension, and right side bend of the spine but was otherwise benign. Conservative management was recommended with follow-up after obtaining lumbar MRI. Upon 
review of imaging, a right sided $8 \times 6 \times 9 \mathrm{~mm}$ synovial cyst exerting a mass effect of the central canal was noted at L4/L5 (Figure 1(a) and Figure 2(a)). After L4/L5 transforaminal epidural steroid injection failed to provide significant relief the patient elected to pursue more definitive treatment.

Patient returned for L4/L5 facet block with cyst aspiration and rupture. Patient preprocedure VAS score was 6/10. Informed consent was obtained. Cyst was identified following contrast injection demonstrating a well circumscribed pattern (Figure 3(a)). $1 \mathrm{cc}$ of synovial fluid was aspirated. Cyst was then ruptured following $4 \mathrm{cc}$ of contrast injection (Figure 3(b)). Patient tolerated the procedure and was discharged home with follow-up for repeat evaluation.

At 8 months post procedure the patient reported improvement in overall LBP for about 3 weeks. His radicular pain had completely resolved, however, his axial lumbar pain had returned recently began to worsen with a VAS 4/10. Repeat MRI showed reduction in the size of the cyst (Figure 1(b) and Figure 2(b)).

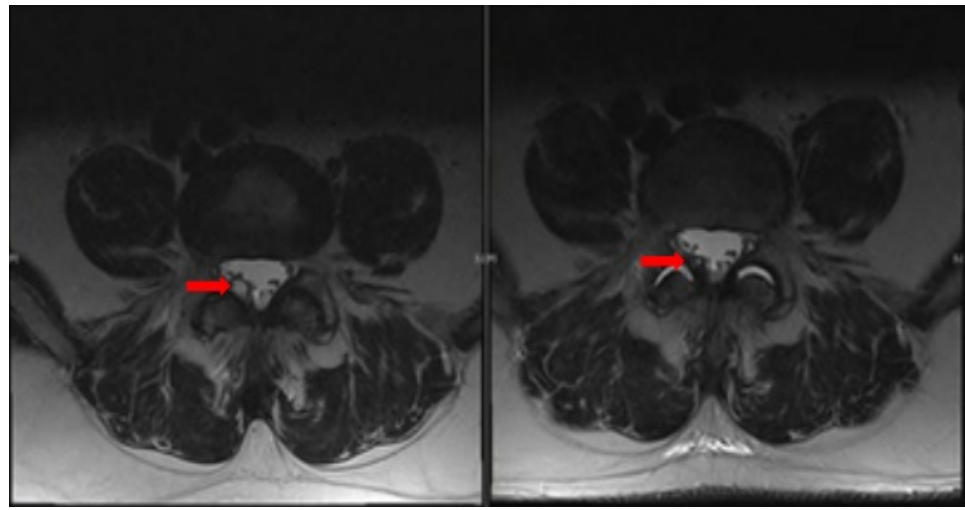

(a)

(b)

Figure 1. (a) T2 weighted axial lumbar MRI showing a facet cyst compressing the L5 nerve root at the L4/L5 spinal level; (b) Follow-up T2 weighted lumbar MRI demonstrating resolution of cyst.

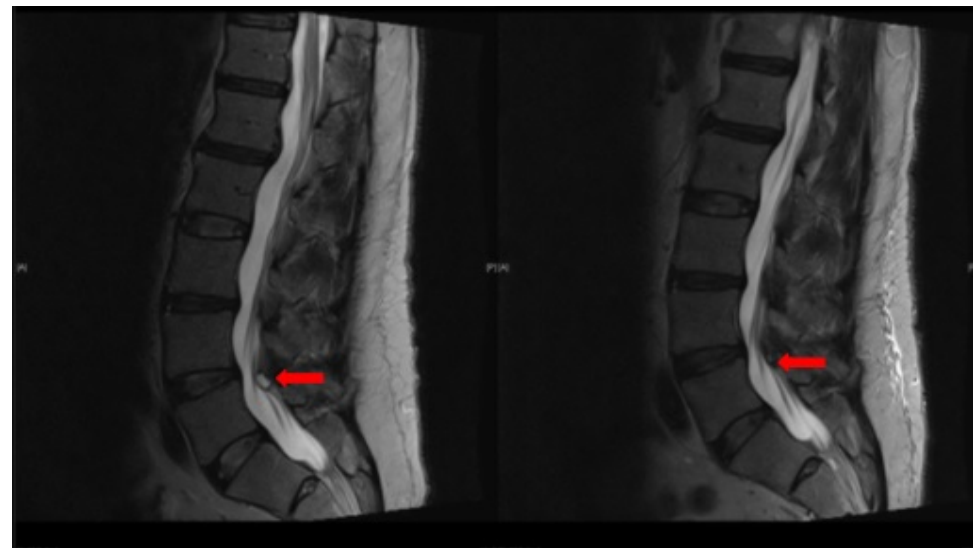

(a)

(b)

Figure 2. (a) T2 weighted sagittal lumbar MRI showing a facet cyst at the L4/L5 spinal level; (b) Follow-up T2 weighted sagittal lumbar MRI demonstrating resolution of cyst. 


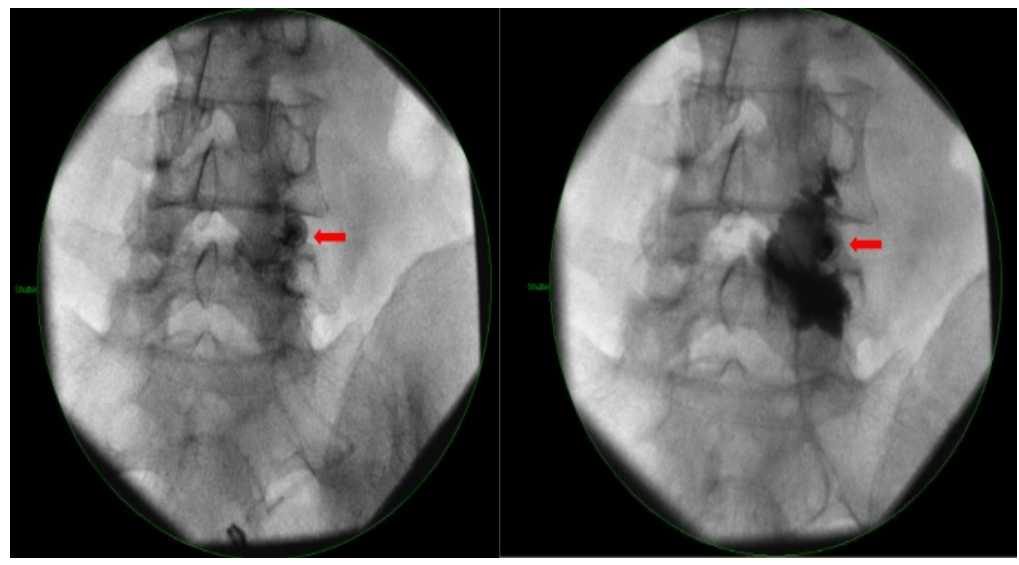

(a)

(b)

Figure 3. (a) Fluoroscopic imaging identifying cyst following injection of contrast; (b) Fluoroscopic imaging demonstrating cyst rupture.

\section{Discussion}

FJ synovial cysts as a cause of LBP with radicular symptoms is an uncommon finding. The overall incidence of anterior facet synovial cysts is reported to be $2.3 \%$ [4]. The incidence of facet joint synovial cysts is increasing due to greater availability of imaging modalities such as MRI and an increase in the aging population. The pathophysiology thought to be due to continued microinstability of the joint, degenerative changes, and repetitive microtrauma of the zygapophyseal joints. The lumbar spine, particularly L4/L5, is the most common location for synovial cyst formation due to increased range of motion of the vertebrate, lordotic curvature and transition in most patients at this level [10]. Not surprisingly, this level is also the most common site for spondylolisthesis [11]. The radicular pain likely is due to direct compression on the nerve roots from the cyst and the axial LBP is caused by persistent facet arthropathy. Conservative treatment is the usual treatment modaliity in uncomplicated cases. If continued pain or radicular symptoms are present, further intervention such as epidural steriod injection, intra-articular injection, cyst aspiration or rupture, and surgical intervention is warrented. The precise intervention is a matter of debate depending on individual patient characteristics and symptoms. In cases of more severe symptoms, radiculopathy or neurogenic claudication, surgical intervention may be elected [3].

Several case studies have demonstrated a consistent finding of degenerative disk disease and osteoarthritic changes in most patients found to have a facet joint synovial cyst [12] [13]. Our patient was in his 6th decade with evidence of facet arthropathy and joint instability which was likely the leading cause of his continued pain and cyst formation. In a retrospective analysis by Bureau et al., twelve patients with facet synovial cysts undergoing percutaneous facet cyst steroid injection, $75 \%$ of patients had excellent relief. Of these, six (67\%) had complete resolution of the cyst and two (22\%) had a reduction in cyst volume on follow-up imaging. The study defined excellent relief as absence of radicular 
symptoms and diminished or absence LBP but did not detail or quantify the degree of LBP relief in those patients. Based on our patient and literature review improvement in radicular pain can be expected with a high probability following complete cyst rupture or diminished cyst volume, however, improvements in axial pain may be transient as cyst steroid injection and rupture does not directly treat the underlying pathology of facet arthropathy. Additionally, 3/12 (25\%) patients in the Bureau study who failed percutaneous rupture with little to no reduction of cyst volume at one-six month follow-up, had only transient or no improvement in symptoms and were referred for surgical cyst removal. While they reported resolution of radicular symptoms following surgical intervention, patients continued to have axial pain [14]. This supports the assertion that reduction in cyst size, either through percutaneous or surgical methods can be expected to improve radicular symptoms, however, axial pain may persist.

Recurrence is a well known outcome of FJ synovial cyst rupture occurring in about $33 \%$ of patients [12]. In a case series involving 101 patients by Martha et al., they demonstrated an increased success rate up to $50 \%$ with repeat rupture [1]. Given the increased risks of complications, morbidity and mortality associated with spinal surgery, repeat cyst rupture may be a better option for certain patient populations. Additionally, the results of the case series found no significant differences in the outcome of current pain in patients who underwent injections and those who received surgical treatment [1]. This suggest that certain patients may require multiple facet cyst steroid injections if they continue to have radicular symptoms and a follow-up MRI demonstrating continued cyst presence of unchanged or only slightly diminished volume. In the study by Bureau et al., 5/12 (42\%) achieved excellent relief after only one facet cyst steroid injection and $7 / 12(58 \%)$ required at least two facet cyst steroid injections. Of these 4/7 (57\%) achieved excellent relief [14]. This is consistent with the research by Martha et al. that repeat injection will increase the success rate up to 50\% [1]. Thus depending on the clinical presentation, presence of radicular symptoms or isolated persistent axial LBP, patients may require either repeat facet cyst rupture or FJ steroid injection respectively.

\section{Conclusions}

FJ synovial cysts are a common cause of back pain which may or may not present with radicular symptoms. The etiology is thought to be due to degenerative processes, microinstability, and repetitive microtrauma. Patients in their 6 th decade of life are at highest risk for development of FJ cysts. The imaging modality of choice for diagnosis is MRI. Treatment options include conservative management including physical therapy, cyst aspiration or rupture, and surgical intervention. Cyst rupture should be considered in patients who are at higher risk for surgical complications or those who would like to avoid surgery. Additionally, repeat cyst rupture can be considered in patients with continued radicular symptoms and evidence of cyst persistence on follow-up MRI.

Our case highlighted the outcome of a patient with severe facet arthropathy 
complicated by a symptomatic lumbar synovial cyst. Although his VAS score had improved and radicular symptoms completely resolved following cyst rupture, the patient still returned requesting additional treatment for continued axial pain. Clinicians performing cyst rupture for radicular symptoms should have a thorough discussion of risks and benefits including the persistence of axial LBP. Further research is needed with longer follow-up periods to determine the persistence of axial LBP after cyst rupture.

\section{References}

[1] Martha, J.F., Swaim, B., Wang, D.A., Kim, D.H., Hill, J., Bode, R. and Schwartz, C.E. (2009) Outcome of Percutaneous Rupture of Lumbar Synovial Cysts: A Case Series of 101 Patients. The Spine Journal: Official Journal of the North American Spine Society, 9, 899-904. https://doi.org/10.1016/j.spinee.2009.06.010

[2] Acharya, R., Patwardhan, R.V., Smith, D.R., Willis, B.K., Fowler, M. and Nanda, A. (2006) Intraspinal Synovial Cysts: A Retrospective Study. Neurology India, 54, 38-41. https://doi.org/10.4103/0028-3886.24700

[3] Boviatsis, E.J., Stavrinou, L.C., Kouyialis, A.T., Gavra, M.M., Stavrinou, P.C., Themistokleous, M. and Sakas, D.E. (2008) Spinal Synovial Cysts: Pathogenesis, Diagnosis and surgical Treatment in a Series of Seven Cases and literature Review. European Spine Journal: Official Publication of the European Spine Society, the European Spinal Deformity Society, and the European Section of the Cervical Spine Research Society, 17, 831-837. https://doi.org/10.1007/s00586-007-0563-Z

[4] Kalevski, S.K., Haritonov, D.G. and Peev, N.A. (2017) Lumbar Intraforaminal Synovial Cyst in Young Adulthood: Case Report and Review of the Literature. Global Spine Journal, 4, 191-196. https://doi.org/10.1055/s-0034-1370694

[5] Kao, C.C., Winkler, S.S. and Turner, J.H. (1974) Synovial Cyst of Spinal Facet. Case Report. Journal of Neurosurgery, 41, 372-376. https://doi.org/10.3171/jns.1974.41.3.0372

[6] Ortiz, A.O. and Tekchandani, L. (2014) Improved Outcomes with Direct Percutaneous CT Guided Lumbar Synovial Cyst Treatment: Advanced Approaches and Techniques. Journal of Neurointerventional Surgery, 6, 790-794. https://doi.org/10.1136/neurintsurg-2013-010891

[7] Gellhorn, A.C., Katz, J.N. and Suri, P. (2013) Osteoarthritis of the Spine: The Facet Joints. Nature Reviews. Rheumatology, 9, 216-224. https://doi.org/10.1038/nrrheum.2012.199

[8] Kalichman, L., Kim, D.H., Li, L., Guermazi, A. and Hunter, D.J. (2010) Computed Tomography-Evaluated Features of Spinal Degeneration: Prevalence, Intercorrelation, and Association with Self-Reported Low Back Pain. The Spine Journal: Official Journal of the North American Spine Society, 10, 200-208. https://doi.org/10.1016/j.spinee.2009.10.018

[9] Ishii, K., Matsumoto, M., Watanabe, K., Nakamura, M., Chiba, K. and Toyama, Y. (2005) Endoscopic Resection of Cystic Lesions in the lumbar Spinal Canal: A Report of Two Cases. Minimally Invasive Neurosurgery: MIN, 48, 240-243. https://doi.org/10.1055/s-2005-870927

[10] Ayberk, G., Ozveren, F., Gok, B., Yazgan, A., Tosun, H., Seckin, Z. and Altundal, N. (2008) Lumbar Synovial Cysts: Experience with Nine Cases. Neurologia Medico-Chirurgica, 48, 298-303; Discussion 303. https://doi.org/10.2176/nmc.48.298 
[11] Deinsberger, R., Kinn, E. and Ungersbock, K. (2006) Microsurgical Treatment of Juxta Facet Cysts of the Lumbar Spine. Journal of Spinal Disorders \& Techniques, 19, 155-160. https://doi.org/10.1097/01.bsd.0000188660.31212.83

[12] Allen, T.L., Tatli, Y. and Lutz, G.E. (2009) Fluoroscopic Percutaneous Lumbar Zygapophyseal Joint Cyst Rupture: A Clinical Outcome Study. The Spine Journal: Official Journal of the North American Spine Society, 9, 387-395.

https://doi.org/10.1016/j.spinee.2008.08.008

[13] Lyons, M.K., Atkinson, J.L., Wharen, R.E., Deen, H.G., Zimmerman, R.S. and Lemens, S.M. (2000) Surgical Evaluation and Management of lumbar Synovial Cysts: The Mayo Clinic Experience. Journal of Neurosurgery, 93, 53-57.

https://doi.org/10.3171/spi.2000.93.1.0053

[14] Bureau, N.J., Kaplan, P.A. and Dussault, R.G. (2001) Lumbar Facet Joint Synovial Cyst: Percutaneous Treatment with Steroid Injections and Distention-Clinical and Imaging Follow-Up in 12 Patients. Radiology, 221, 179-185.

https://doi.org/10.1148/radiol.2211010213 